\title{
Hydrocephalus in a newborn (2009: 7a)
}

(C) European Society of Radiology 2008

\section{Case report}

A male newborn was delivered by caesarean section due to cephalopelvic disproportion. The APGAR scores were 8 and 9 at 1 and $5 \mathrm{~min}$, respectively. Clinical examination revealed sutural diasthesis, a head circumference of $45 \mathrm{~cm}$ (above the 95th percentile for age), hypotonia and weak spontaneous motor activity. The child kept his hands preferably closed, with clasped thumbs.

Brain MRI performed on the second day of life showed severe supratentorial hydrocephalus (Figs. 1 and 2).

\section{What is the diagnosis?}

Readers are invited to supply one possible diagnosis via electronic means to: robert.hermans@uzleuven.be
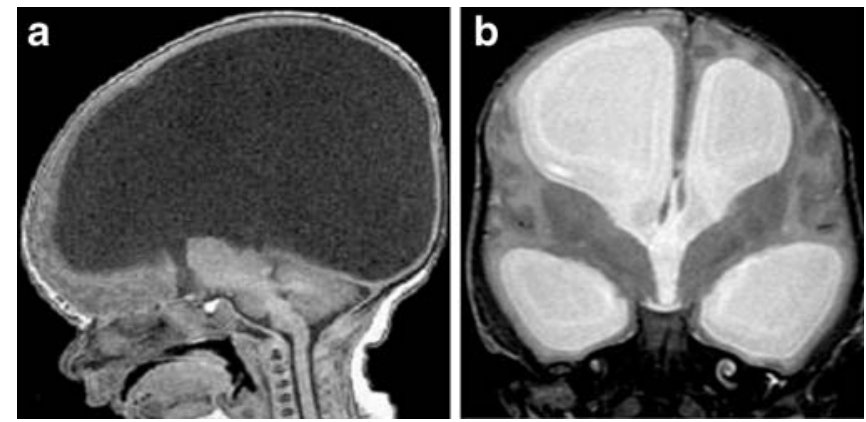

Fig. 1 a,b Brain MRI performed on the 2nd day of life. a Sagital T1WI, b Coronal T2WI
The subject of the email should include 'Interpretation Corner' and the number 2009: 7a.

You should include your name, title, address, fax and phone number.

Deadline: one clear calendar month from distribution date.

Three months after the initial publication of the case history, the authors will publish the final diagnosis and a brief summary. The summary will describe exactly how the case was investigated at the host institution, how the diagnosis was established and the teaching points of the case in question.

The names of the first 25 radiologists submitting the correct diagnosis will be published (only one from any individual centre and none from the host institution!).
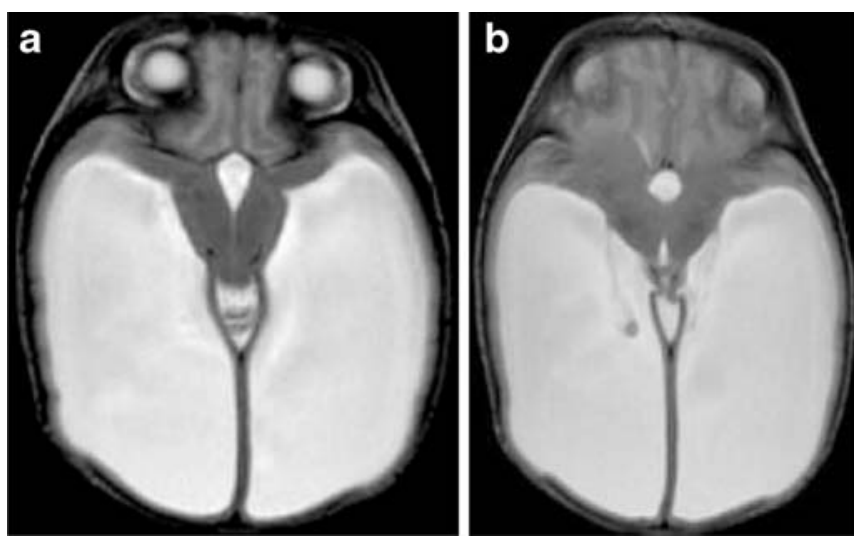

Fig. 2 a,b Brain MRI performed on the 2nd day of life. a and $\mathbf{b}$ Axial T2WI 